\title{
Iron related donor-like defect in zinc oxide
}

V. Quemener, , L. Vines, , E. V. Monakhov, and, and B. G. Svensson

Citation: Appl. Phys. Lett. 102, 232102 (2013); doi: 10.1063/1.4809941

View online: http://dx.doi.org/10.1063/1.4809941

View Table of Contents: http://aip.scitation.org/toc/apl/102/23

Published by the American Institute of Physics

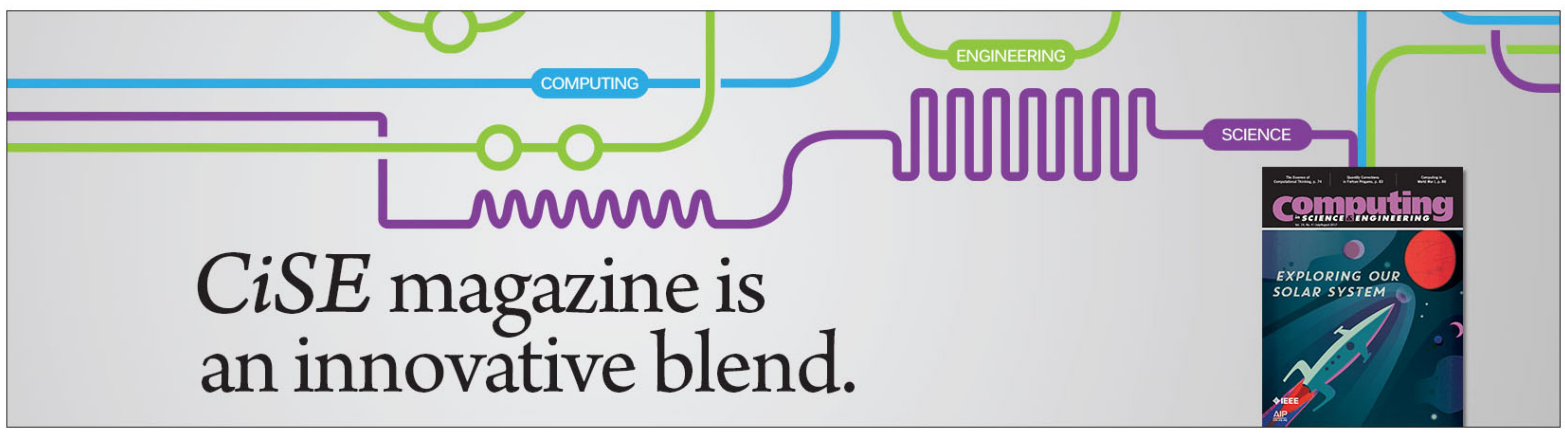




\title{
Iron related donor-like defect in zinc oxide
}

\author{
V. Quemener, ${ }^{\text {a) }}$ L. Vines, E. V. Monakhov, and B. G. Svensson \\ Department of Physics/Center for Materials Science and Nanotechnology, University of Oslo, \\ P.O. Box 1048 Blindern, N-0316 Oslo, Norway
}

(Received 2 May 2013; accepted 25 May 2013; published online 10 June 2013)

\begin{abstract}
Hydrothermally grown $\mathrm{ZnO}$ samples, annealed in $\mathrm{Zn}$-rich and O-rich ambients, have been investigated by deep level transient spectroscopy. A clear correlation has been found between the annealing treatment and the formation/suppression of two defect levels at $\sim 0.19$ (E2) and $\sim 0.54$ (E4) $\mathrm{eV}$ below the conduction band edge $\left(E_{c}\right)$. Moreover, a close proportionality over more than three orders of magnitude has been established between the concentration of E2 and that of $\mathrm{Fe}$, as determined by secondary ion mass spectrometry. Based on the above observations and previous reports in the literature, Fe on Zn-site is a likely candidate for E2. (C) 2013 AIP Publishing LLC. [http://dx.doi.org/10.1063/1.4809941]
\end{abstract}

The control and understanding of native and impurityrelated defects in zinc oxide $(\mathrm{ZnO})$ represents one of the major challenges in order to implement $\mathrm{ZnO}$ in future devices such as solar cells and light emitting diodes. From the growth process and the device processing, intrinsic and impurity-related defects are readily present in the material. They can play an important role for the native n-type conduction and the difficulty in achieving reliable p-type conduction. So far, defects in the band gap have been identified both by optical and electrical characterization techniques and reported in the literature. In particular, the upper part of the band gap has been extensively investigated by capacitance spectroscopy techniques and at least 8 defect levels have been reported. ${ }^{1-6}$ Despite several attempts to identify the origin of these defects, no consensus has been reached so far, and therefore, further investigations are needed to determine their identities. Moreover, impurities are expected to play an important role in the formation of several of the reported defect levels. For instance, $\mathrm{Al}$ and $\mathrm{H}$ demonstrate unambiguously their roles in the conductivity with shallow levels close to the conduction band edge $\left(\mathrm{E}_{c}\right){ }^{7-9}$ However, the role of common impurities for deep levels is much less known and data remain scarce in the literature.

Post-growth annealing under controlled ambient conditions can modify the equilibrium concentration of deep level defects and the effects can be monitored by deep level transient spectroscopy (DLTS) measurements. This can provide indications on the origin of the modified defect levels. Several defects show strong correlation with the annealing conditions, where defects can be manipulated by, e.g., annealing under zinc-rich and oxygen-rich conditions. ${ }^{2,5,10} \mathrm{In}$ particular, a clear correlation occurs between annealing in $\mathrm{Zn}$ ambient and the formation of a level at $\sim 0.54 \mathrm{eV}$ below $\mathrm{E}_{c}$, and the suppression of a defect level at $\sim 0.19 \mathrm{eV}$ below $\mathrm{E}_{c}$. Interestingly, these two levels behave in the opposite way after annealing under O-rich conditions, as reported in Ref. 5.

In the present work, we correlate the evolution of deeplevel defects in hydrothermally grown $\mathrm{ZnO}(\mathrm{HT}-\mathrm{ZnO})$ samples after annealing in $\mathrm{Zn}$ - and O-rich ambients with chemical impurity analysis using secondary ion mass spectrometry (SIMS).

a)Electronic mail: vincent.quemener@smn.uio.no
In particular, the concentration of the $E_{c}-0.19 \mathrm{eV}$ level, formed under O-rich conditions, shows a proportionality with the $\mathrm{Fe}$ content over more than three orders of magnitude, strongly suggesting that the level is iron related.

The samples used were cut from hydrothermally grown n-type $\mathrm{ZnO}$ bulk wafers (thickness of $\sim 0.5 \mathrm{~mm}$ ) with orientation perpendicular to the c-axis ([0001] direction), purchased from Tokyo Denpa (sample A) and SPC Goodwill (samples B and C). Sample A (with a resistivity of $50 \Omega \mathrm{cm}$ ) was first heat treated at $1100^{\circ} \mathrm{C}$ for $30 \mathrm{~min}$ in a sealed quartz ampoule holding $99.99 \% \mathrm{Zn}$ metal (labelled A1) and then annealed at $1100^{\circ} \mathrm{C}$ during $1 \mathrm{~h}$ in flowing oxygen atmosphere (labelled A2). A similar process was performed for the samples B and C, except that both were pre-annealed at $1400^{\circ} \mathrm{C}$ during $1 \mathrm{~h}$ in $\mathrm{Ar}$ ambient, before the $\mathrm{O}$ - and $\mathrm{Zn}$-treatment. After each annealing treatment, both O- and $\mathrm{Zn}$ - terminated surfaces were mechanically polished on a rotating disc with diamond slurry using different grain sizes from $6 \mu \mathrm{m}$ to $0.25 \mu \mathrm{m}$, which removes a layer with a total thickness of about $20-30 \mu \mathrm{m}$ on each side. The samples were finally etched in a $2 \%$ hydrofluoric acid solution $(H F)$ for $2 \mathrm{~min}$, in order to minimize the amount of interface defects formed by the polishing. ${ }^{11}$ Before Schottky contact deposition, the samples were cleaned in acetone $(5 \mathrm{~min})$ and isopropanol $(5 \mathrm{~min})$ in an ultrasonic bath and then treated in a boiling $40 \%$ hydrogen peroxide $\left(\mathrm{H}_{2} \mathrm{O}_{2}\right)$ solution for $1 \mathrm{~min} .100 \mathrm{~nm}$ thick Palladium (Pd) Schottky contacts, with a diameter of $0.30 \pm 0.01 \mathrm{~mm}, 0.50 \pm 0.01 \mathrm{~mm}$ and $0.80 \pm 0.01 \mathrm{~mm}$, were deposited on the $\mathrm{Zn}$-polar face by electron beam evaporation of a $99.999 \%$ pure Pd source through a molybdenum shadow mask at a base pressure of $10^{-6}$ mbar. Silver paste was used as Ohmic contact on the back side (O-face).

Current-voltage (I-V) and capacitance-voltage (C-V) measurements were performed in darkness at room temperature using a Keithley 617 electrometer and a $1 \mathrm{MHz}$ capacitance meter (HP $4280 \mathrm{~A}$ ), respectively. The I-V results showed stable rectifying behavior of about 3-4 orders of magnitudes for all the contacts on each sample. The DLTS measurements were performed in the temperature range of $70 \mathrm{~K}-300 \mathrm{~K}$ with a reverse bias voltage of $-3 \mathrm{~V}$ and a pulse voltage of $3 \mathrm{~V}$ ( $50 \mathrm{~ms}$ duration) using a refined version of the setup described in Ref. 12. The DLTS signal was extracted 
applying a lock-in weighting function with different rate windows in the range $(20 \mathrm{~ms})^{-1}$ to $(640 \mathrm{~ms})^{-1}$. The chemical characterization was carried out by SIMS using a Cameca IMS 7f instrument with a primary beam of $10 \mathrm{keV} \mathrm{O}_{2}^{+}$or $15 \mathrm{keVCs}^{+}$ions. The impurity concentrations were calibrated using implanted reference samples. The crater depths were measured with a Dektak 8 stylus profilometer and a constant erosion rate as a function of sputtering time was assumed.

Figure 1(a) shows DLTS spectra of sample A in the asgrown state and after the different annealing treatments. Three main levels appear in the $80-300 \mathrm{~K}$ range, labelled E2, $\mathrm{E} 3$, and $\mathrm{E} 4$, with energy level positions of $\sim E_{c}-0.19$, $\sim E_{c}-0.30$, and $\sim E_{c}-0.54 \mathrm{eV}$, respectively. These levels are commonly observed in hydrothermally grown samples treated at high temperatures, ${ }^{1,2,4,5}$ and the annealing conditions influence considerably their amplitudes, in agreement with previous results. ${ }^{2,5}$ In the as-grown state $(\mathrm{A} 0)$, only E3 is present. Annealing in $\mathrm{Zn}$-rich ambient gives rise to E4. On the other hand, after annealing in O-rich ambient, E4 disappears and E2 emerges, having a low concentration close to the detection limit of the measurements. Similar results for the E2 and E4 levels are also observed in samples B (Fig. 1(b)) and C (not shown), and they are consistent with those previously reported in Ref. 5 .

The E3 level is much less affected by the treatments in the $\mathrm{Zn}$ - and O-rich ambients than E2 and E4, and E3 remains
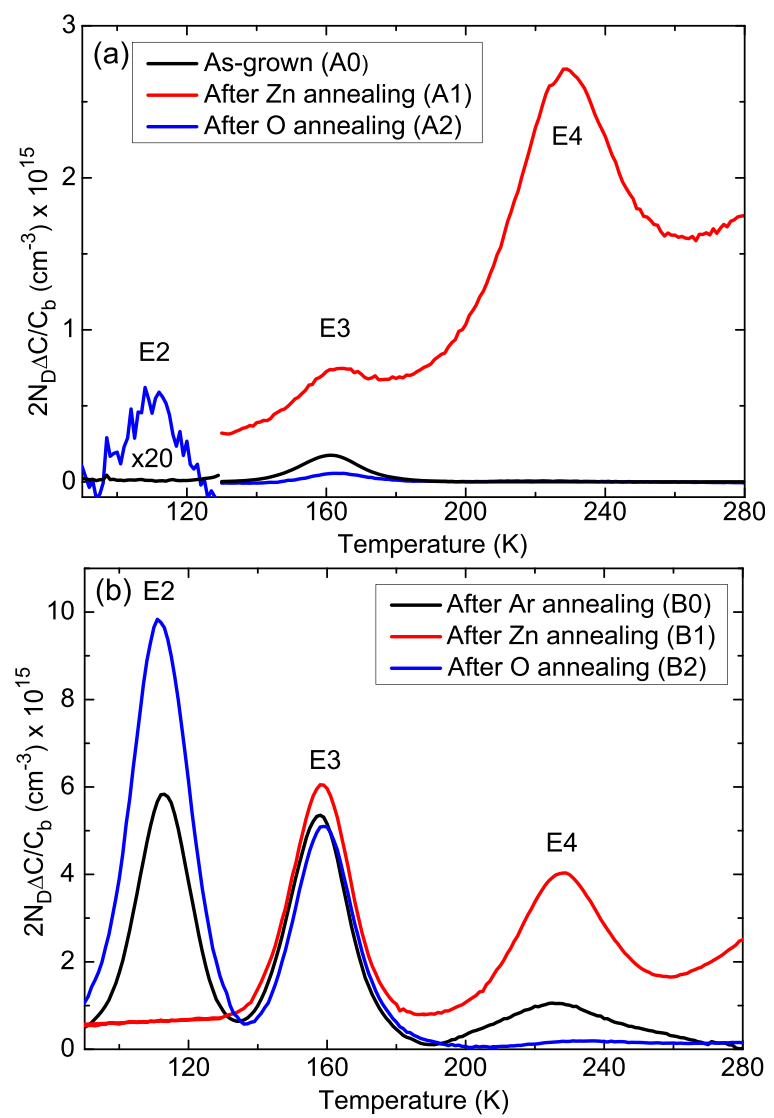

FIG. 1. DLTS spectra from samples A (a) and B (b) after different annealing processes using a rate window of $(640 \mathrm{~ms})^{-1}$. A reverse bias voltage of $-3 \mathrm{~V}$ and filling pulse voltage of $3 \mathrm{~V}$ have been employed. $\mathrm{C}_{b}$ represents the reverse bias capacitance, $\mathrm{N}_{D}$ is the carrier concentration, and $\Delta C$ is the amplitude of the capacitance transient. essentially constant after the Zn-treatment when correcting for the increase in background signal of sample A1. In sample A, after the O-treatment, E3 is reduced by a factor of two relative to the as-grown state; but in absolute values, this corresponds only to a reduction by $\sim 1 \times 10^{14} \mathrm{~cm}^{-3}$. In Ref. 5 where samples having an absolute concentration of $\sim 50-500$ times higher than that in sample $\mathrm{A}$, the relative reduction after O-treatment was small and essentially within the accuracy of the measurements. Hence, the present results corroborate the previous ones reported in the literature, showing that annealing treatments in different ambients up to $\sim 1200{ }^{\circ} \mathrm{C}$, electron and ion irradiation/implantation, and polishing have limited influence on the absolute concentration of $\mathrm{E} 3,{ }^{4-6,11,13,14}$ and its identity remains puzzling. However, the large variation in absolute concentration values between different samples, subjected to similar (equilibrium) annealing conditions, and very limited influence by $\mathrm{O}$ - or Zn-rich ambients, indicate an impurity-related center rather than an intrinsic one. This conclusion is also substantiated by the data in Fig. 2, revealing a strong increase of the E3 concentration in the samples with a generally high impurity content, although no linear correlation could be established with any of the specific impurities monitored. Moreover, as suggested in Ref. 15, E3 can arise from two closely defect levels where one (dominant in sample A) can be more affected by the annealing conditions than the other.

Also E2 and E4 are included in Fig. 2 and compared with the concentration of typical impurities in the three different samples (as measured by SIMS); generally, sample A exhibits much lower values compared to those of samples B and $\mathrm{C}$, where $\mathrm{Al}, \mathrm{Mn}$, and $\mathrm{Fe}$ are found to be about two orders of magnitude lower in A than in B and C. The difference between samples B and C is smaller but B contains in general lower levels of contamination compared to $\mathrm{C}$ (except for $\mathrm{Li}$ ) by almost one order of magnitude in some cases. The concentration values of E2 and E4 represent those after annealing in O-rich and $\mathrm{Zn}$-rich ambients, respectively, and are also given in Table I. The concentration of E4 remains essentially independent on the origin of the sample and hinges only on the annealing conditions, in agreement with previous results. ${ }^{2,5}$ Hence, these findings corroborate an assignment of $\mathrm{E} 4$ to a defect of intrinsic nature promoted by Zn-rich conditions. ${ }^{5}$

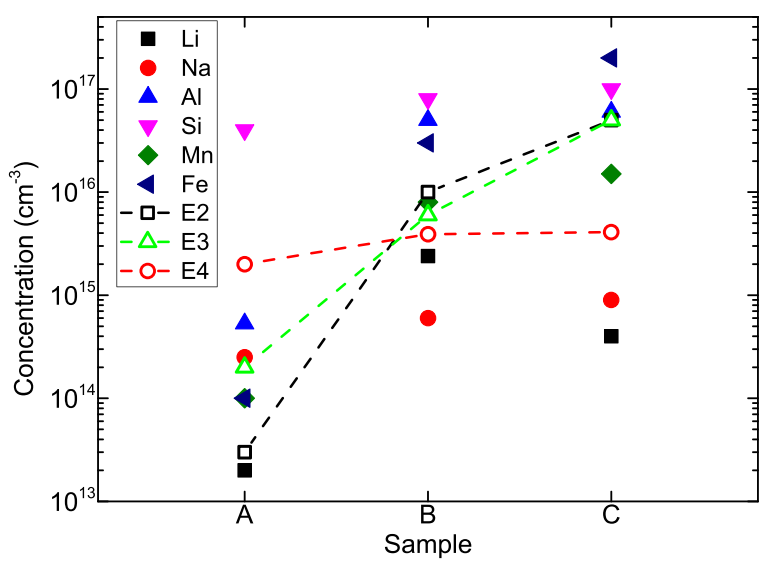

FIG. 2. Measured impurity concentrations using SIMS and the concentrations of E2, E3, and E4 for the three different samples (A, B, and C). 
TABLE I. E2 and E4 concentrations after O-rich and Zn-rich annealing treatment, respectively, for the samples A, B, and C.

\begin{tabular}{lll}
\hline \hline Sample & E2 $\left(\mathrm{cm}^{-3}\right)$ & E4 $\left(\mathrm{cm}^{-3}\right)$ \\
\hline A & $3 \times 10^{13}$ & $2 \times 10^{15}$ \\
B & $1 \times 10^{16}$ & $4 \times 10^{15}$ \\
C & $5 \times 10^{16}$ & $4 \times 10^{15}$ \\
\hline \hline
\end{tabular}

In direct contrast to E4, a large difference in the E2 concentration occurs for the three samples. Assuming that steady state condition is reached during the heat treatment in O-rich ambient, an intrinsic nature of E2 can be excluded. Moreover, E2 is not consistently reported in the literature and its presence depends strongly on the $\mathrm{ZnO}$ growth conditions. ${ }^{2,13,16,17}$ This implies that E2 is due to an impurity related defect, and indeed, the concentration of E2 follows closely that of iron in the three different samples, where E2 represents about $30 \%$ of the total $\mathrm{Fe}$ concentration in each sample. This proportionality is illustrated in Figure 3 and holds over a dynamic range of three orders of magnitude. This is a strong evidence that E2 involves Fe in a configuration enhanced by O-rich conditions, such as $\mathrm{Fe}$ on $\mathrm{Zn}$ site. It should also be mentioned that the Fe concentration remains constant in all the samples after the different annealing treatments, as determined by SIMS (not shown). This may indicate a high migration energy of $\mathrm{Fe}$ and a lower binding energy of E2 which would explain a change in the defect configuration with the annealing conditions (activation under O-rich conditions and deactivation under Zn-rich conditions), while the $\mathrm{Fe}$ concentration is maintained.

Recently, Jiang et al. ${ }^{18}$ have studied transition metals in $\mathrm{ZnO}$ using electron paramagnetic resonance measurements. In their study, the ionization energy for the substitutional $\mathrm{Fe}$ donor $\left(F e_{Z n}^{2+}\right)$ has been estimated as $\sim 0.24 \mathrm{eV}$ with an uncertainty of $\pm 0.05 \mathrm{eV}$. Jiang et al. have related either $F e_{Z n}^{2+}$ or $N i_{Z n}^{2+}$ to the dominant E3 level, commonly observed in DLTS spectra, adding to the controversy on its identification. Our results show that assignment of $\mathrm{Fe}_{\mathrm{Zn}}^{2+}$ to E2 can be more substantiated: the energy position of E2, its donor-like behavior, ${ }^{5}$ and the response on annealing ambient.

In summary, hydrothermally grown $\mathrm{ZnO}$ samples have been sequentially annealed under $\mathrm{Zn}$ and O-rich conditions. Strong correlations have been found between the annealing treatments and the formation/suppression of the E2 $\left(E_{c}-0.19 \mathrm{eV}\right)$ and $\mathrm{E} 4\left(E_{c}-0.54 \mathrm{eV}\right)$ levels. It is found that E2 can be ascribed to a Fe-related defect promoted by O-rich conditions. The concentrations of E2 and Fe exhibit a close proportionality over a range of three orders of magnitude, where E2 amounts to $\sim 30 \%$ of the total Fe concentration, as determined by SIMS. These findings suggest that E2 arises from $\mathrm{Fe}$ on $\mathrm{Zn}$ site, with the $F e_{\mathrm{Zn}}^{2+}$ donor as a likely candidate. $\mathrm{E} 4$, on the other hand, is promoted by Zn-rich conditions and is presumably of intrinsic origin, showing only a dependence on the annealing conditions used irrespective of

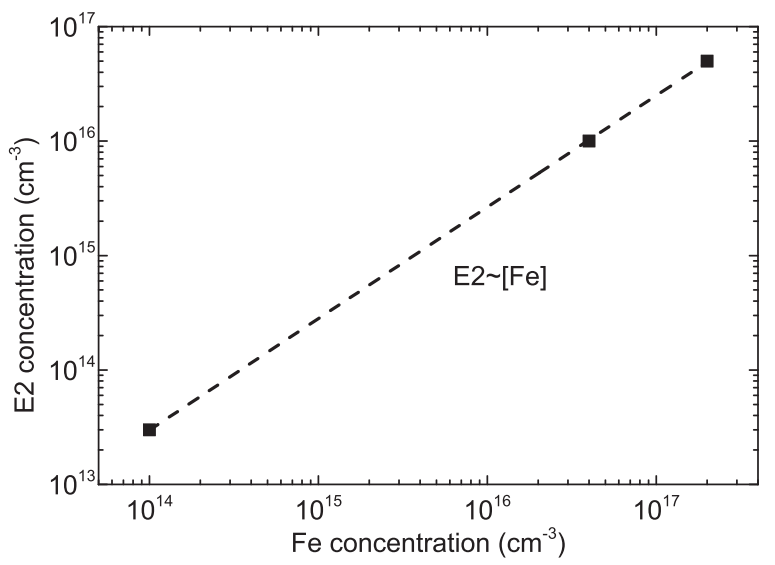

FIG. 3. The E2 concentration after annealing in oxygen as a function of the total Fe concentration. The fitted curve indicates that [E2] increases proportionally with $[\mathrm{Fe}]$ (brackets denote concentration values).

the impurity content in the samples. Finally, it is argued that the commonly observed E3 level is impurity-related and not of intrinsic nature, as frequently assumed in the literature.

This work was supported by the Norwegian Research Council through the NanoMat (CONE Project) and FRIENERGI Programs.

${ }^{1}$ F. D. Auret, S. A. Goodman, M. J. Legodi, W. E. Meyer, and D. C. Look, Appl. Phys. Lett. 80, 1340 (2002).

${ }^{2}$ T. Frank, G. Pensl, R. Tena-Zaera, J. Zúniga-Pérez, C. Martínez-Tomás, V. Munoz-Sanjosé, T. Ohshima, H. Itoh, D. Hofmann, D. Pfisterer, J. Sann, and B. Meyer, Appl. Phys. A 88, 141-145 (2007).

${ }^{3}$ R. Schifano, E. V. Monakhov, B. G. Svensson, W. Mtangi, P. Janse van Rensburg, and F. D. Auret, Physica B 404, 4344-4348 (2009).

${ }^{4}$ L. Vines, E. V. Monakhov, and B. G. Svensson, Physica B 404, 4386 (2009).

${ }^{5}$ V. Quemener, L. Vines, E. V. Monakhov, and B. G. Svensson, Appl. Phys. Lett. 100, 112108 (2012).

${ }^{6}$ L. Vines, J. Wong-Leung, C. Jagadish, V. Quemener, E. V. Monakhov, and B. G. Svensson, Appl. Phys. Lett. 100(21), 212106 (2012).

${ }^{7}$ F. A. Selim, M. H. Weber, D. Solodovnikov, and K. G. Lynn, Phys. Rev. Lett. 99, 085502 (2007).

${ }^{8}$ E. V. Monakhov, A. Yu Kuznetsov, and B. G. Svensson, J. Phys. D: Appl. Phys. 42, 153001 (2009).

${ }^{9}$ M. H. Weber and K. G. Lynn, J. Phys. Conf. Ser. 262, 012063 (2011).

${ }^{10}$ D. M. Hofmann, D. Pfisterer, J. Sann, B. K. Meyer, R. Tena-Zaera, V. Munoz-Sanjosé, T. Frank, and G. Pensl, Appl. Phys. A 88, 147 (2007).

${ }^{11}$ V. Quemener, L. Vines, E. V. Monakhov, and B. G. Svensson, Appl. Phys. Lett. 99, 112112 (2011).

${ }^{12}$ B. G. Svensson, K.-H. Rydén, and B. M. S. Lewerentz, J. Appl. Phys. 66, 1699 (1989).

${ }^{13}$ F. D. Auret, S. A. Goodman, M. Hayes, M. J. Legodi, H. A. van Laarhoven, and D. C. Look, Appl. Phys. Lett. 79(19), 3074 (2001).

${ }^{14}$ L. Vines, J. Wong-Leung, C. Jagadish, E. V. Monakhov, and B. G. Svensson, Physica B 407, 1481 (2012).

${ }^{15}$ H. von Wenckstern, H. Schmidt, M. Grundmann, M. W. Allen, P. Miller, R. J. Reeves, and S. M. Durbin, Appl. Phys. Lett. 91, 022913 (2007).

${ }^{16}$ M. Hayes, F. D. Auret, P. J. Janse van Rensburg, J. M. Nel, W. Wesch, and E. Wendler, Nucl. Instrum. Methods Phys. Res. B 257, 311 (2007).

${ }^{17}$ L. Scheffler, V. 1. Kolkovsky, E. V. Lavrov, and J. Weber, J. Phys.: Condens. Matter 23, 334208 (2011).

${ }^{18}$ Y. Jiang, N. C. Giles, and L. E. Halliburton, J. Appl. Phys. 101, 093706 (2007). 